\title{
The Critical Current and Normal Resistance of High-Tc Step-Edge SNS Junctions
}

\author{
C.D. Reintsema, R.H. Ono, G. Barnes, L. Borcherdt, T.E. Harvey, G. Kunkel, D.A. Rudman, and L.R. Vale \\ Electromagnetic Technology Division, NIST, Boulder CO \\ N. Missert \\ Sandia National Laboratory, Albuquerque NM
}

P.A. Rosenthal

Advanced Fuel Research, Hartford CT

\begin{abstract}
We have fabricated high-Tc superconductor-normal-superconductor Josephson junctions with a variety of controlled geometries and measured the resulting dependences of critical current and normal resistance. These studies show that we can adjust our junction parameters over orders of magnitude, thus allowing us to tailor the junctions for a variety of applications.
\end{abstract}

\section{INTRODUCTION AND BACKGROUND}

We report here on recent and ongoing work on a particular variety of high-temperature superconducting (HTS) Josephson junction: the step-edge superconductor-normal-superconductor (SNS) weak link [1]-[4] made using $\mathrm{YBa}_{2} \mathrm{Cu}_{3} \mathrm{O}_{7-\mathrm{x}}$ (YBCO) and $\mathrm{Au}$. We have conducted a study of the dependence of junction critical current $I_{c}$ and normal resistance $R_{n}$ on lithographically determined junction dimensions; this study shows that we can vary junction parameters independently and as needed for a variety of applications. This paper will summarize some of the results of this work, and will conclude with a brief discussion of further process development now underway.

Josephson junctions made from HTS thin films can be divided into two broad categories: grain boundary junctions and SNS junctions. The two most heavily studied versions of the SNS variety are the SNS edge junctions (e.g. [5]) and the step-edge SNS junction [1],[2]. We have refined the process for making step-edge SNS junctions, schematic drawings of which are shown in Fig. 1. We have found that it is possible to vary the junction dimensions to adjust both $I_{C}$ and $R_{n}$ independently. In doing this we made junctions with very high $I_{C} R_{n}$ products: over $10 \mathrm{mV}$ at $4 \mathrm{~K}$, and greater than $1 \mathrm{mV}$ at $60 \mathrm{~K}$.

Our junctions can be described macroscopically by the Resistively Shunted Junction (RSJ) model, which requires only $I_{C}, R_{n}$, the junction capacitance $C_{J}$, and the temperature to characterize the dynamics of a Josephson junction. However, this phenomenological model does not address the microscopic origin of any of the junction parameters. In the

Manuscript submitted Oct. 16, 1994.

Contribution of the U.S. Government, not subject to copyright case of SNS junctions, the theoretical description begins with deGennes' treatment of the proximity effect [6]. Likharev used several approximations to develop a microscopic description of transport [7]. These theories still comprise the standard model for analyzing the $I_{C} R_{n}$ product of SNS weak links as a function of material properties and temperature. While temperature dependence is fundamental and important, this paper will also address a less-frequently investigated dependence: that of $I_{c}$ on normal metal length. We present here a preliminary study of length dependence for the case of noble metal barriers.

The proximity effect theory of SNS junctions predicts that the superconducting order parameter decays into the normal metal region in an approximately exponential fashion. Coupling of the order parameter leads to a superconducting critical current and the following expression for $I_{C} R_{n}$ [6]:

$$
I_{c} R_{n}=\frac{2 \Delta^{2}(0)(1-t)^{2}}{\pi e k_{B} T_{c}} \frac{d_{n}}{\xi_{n}} \frac{1}{\sinh \left(d_{n} / \xi_{n}\right)}
$$

where $\Delta(0)$ is the gap energy at $T=0, d_{n}$ is the normal metal length, $t=T / T_{c}$, and the normal metal coherence length is $\xi_{n}=\left(h v_{F} l / 6 \pi k_{B} T\right)^{1 / 2}$ in the dirty limit where $l$ is the mean free path. The issue of clean vs. dirty limit coherence lengths is addressed in [8]. In the case of our noble metal bridges, the difference implied by the two limits is not large. The Likharev calculation [7] adds the concept of a "rigid" boundary condition which acts to discontinuously depress the order parameter at the interface. This alters the temperature and length dependences of $I_{c} R_{n}$, typically reducing the maximum $I_{c} R_{n}$ achievable from the deGennes result. We also note that these theories do not take into account non-uniform current distributions in the junctions.

Varying the junction width, $w$ (see Fig. 1b), affects both the critical current and the normal resistance. The resistance should scale inversely with the width, even with the complication of barrier resistance $R_{B}$. The critical current varies in a fashion determined by an effective Josephson penetration depth, $\lambda_{J}$. In the case of a tunnel junction, $\lambda_{J}=\left(\phi_{0} / 2 \pi \mu_{0} J_{c}\left(2 \lambda_{0}+d\right)\right)^{1 / 2}$ where $\phi_{0}$ is the flux quantum, $\lambda_{0}$ is the London penetration depth, $J_{c}$ is the current density, and $d$ is the barrier thickness. An expression for our planar weak links, derived in [9], is different but still scales inversely with $I_{c}{ }^{1 / 2}$. Junctions narrower than a few $\lambda_{J}$ 
have critical currents that scale linearly with $w$. We expect some degree of non-uniformity in the current distribution in both the banks leading to the junction and in the junction itself. The effect of this has not yet been modeled and remains a subject for future study.

The $R_{n}$ of an SNS junction is comprised of $R_{B}$ at each SN interface and the resistance of the link itself. The resistance of our junctions can only be accounted for by a large $R_{B}$ since the measured resistivity of our Au films range from only $2-4 \mu \Omega-\mathrm{cm}$ between 40 and $100 \mathrm{~K}$; this resistivity predicts a bridge resistance of less than $0.01 \Omega$. It is likely that a S-I-N-I-S model (where I is a dielectric barrier) will prove to be a more accurate predictor of junction behavior [10]. While $I_{C} R_{n}$ is the usual figure of merit for junctions, we have shown that $I_{c}$ and $R_{n}$ can be varied independently in our step-edge SNS junctions [4]. An analysis of the origin of $R_{n}$ in our junctions is presently underway [10],[11].

\section{EXPERIMENTAL DETAILS}

Details of our fabrication processes have been described elsewhere [2]-[4]. The junctions are formed across a step in either the substrate or in a layer of deposited dielectric. The substrates used in this work were single-crystal $\mathrm{LaAlO}_{3}$ (LAO) and $\mathrm{NdGaO}_{3}$ (NGO); they were usually covered with a 200-500 nm thick layer of $\mathrm{SrTiO}_{3}$ (STO) for step formation. Ion milling formed the steps through a photoresist mask. We grow the YBCO film by pulsed laser deposition at an angle which acts to shadow the film growth, thereby leaving a break between the upper and lower film. This is filled by an in situ deposition of $100 \mathrm{~nm}$ of Au from a sputter source. For the first set of length-dependence results we will discuss, the process was modified: three separate steps were ion-milled into the same sample, and arrays of junctions were formed across each step. By varying the milling time for the three different steps we obtain three different step heights. Referring again to Fig. 1, the effective, or average, length of the junction, $L_{e f f}$, decreases with decreasing $d_{\text {step }}$ and increasing $d_{y b c o}$. In all cases, $\mathrm{Ar}$ ion milling was used to pattern the bilayer and subsequent patterning of the normal metal for the "resistor cut" experiments of section III-B.

The layout of our experimental samples uses a $10 \mathrm{~mm}$ die size with 20 contact pads. An advantage of the bilayer growth is that contact pads are automatically Au covered for low contact resistance regardless of the type of contact made; in most cases we have used low magnetic field spring-loaded pins. The arrays used in many of these studies are made simply by meandering the Au-YBCO bilayer across an edge in the STO or the substrate. Each crossing forms a junction, in many cases with individual voltage taps between junctions. For the "terraced" design shown in cross section in Fig. 1c, we meandered arrays across each of the steps, with taps coming off between steps.

Measurements were carried out in an rf-filtered dip-probe in a magnetically shielded liquid-He dewar. Current-voltage $I V$ characteristics and differential resistance $d V / d I$ vs. current were recorded using a computerized data acquisition system. Many of the results we present here were obtained by analyzing the differential resistance of series arrays of junctions. A junction that follows RSJ characteristics will have a sharp peak in $d V / d I$ at the critical current. Maxima are easily seen and measured in series arrays, corresponding to individual junctions switching into the voltage state.
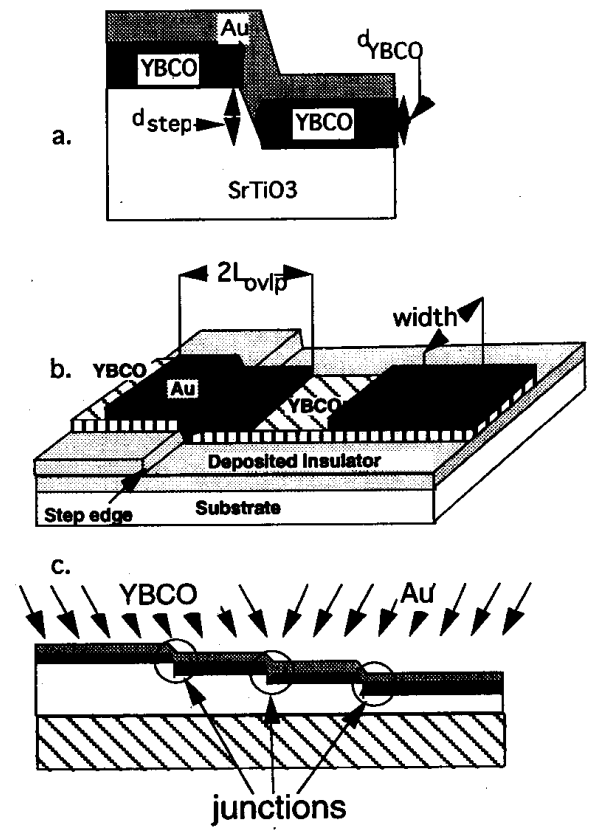

FIG. 1 a. schematic drawing of the step-edge SNS junction, showing the shortest conduction path and its dependence on step height and YBCO thickness. b. drawing of junction showing the width and the effect of Au removal near the junction. c. drawing of the "terraced" junctions used to test the effect of step height on junction characteristics.

\section{RESULTS OF LENGTH AND WIDTH VARIATIONS}

\section{A. Variation of $I_{C}$ with Length and Width}

The variation of $I_{c}$ with junction width, length, and temperature can be compared with theoretical predictions, and various microscopic parameters such as coherence lengths, energy gaps, and penetration depths can be extracted. The difficulty with carrying out such an extraction using HTS junctions has been partly due to the difficulty in separating run-to-run variation of junction properties from effects due to deliberately varied parameters. We have developed a novel fabrication scheme designed to investigate the dependence of $I_{C}$ on the effective length of a step-edge SNS junction by examining arrays of junctions on the same sample, grown in one deposition across three steps of varying height. We have used the terraced step-edge fabrication process to study resulting arrays of varying effective length. The results for one set of junctions are shown in Fig. 2. We plot the currents corresponding to peaks in $d V / d I$ as a function of the step height. As $d_{\text {step }}$ increases for fixed $d_{y b c o}$, junction 
length increases. The data shown are at $4 \mathrm{~K}$.

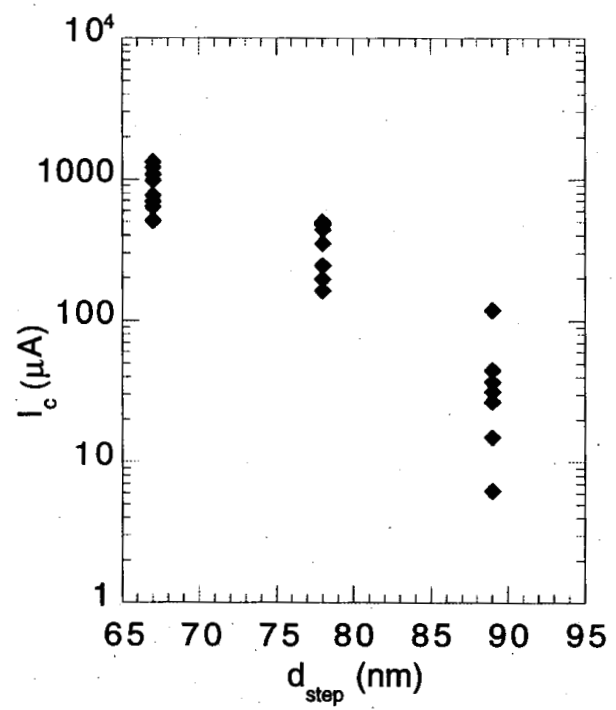

FIG. 2. Dependence of $I_{C}$ on step height. The data are from three series arrays at $4 \mathrm{~K}$, grown and patterned simultaneously on the same sample. The YBCO was $80 \mathrm{~nm}$ thick.

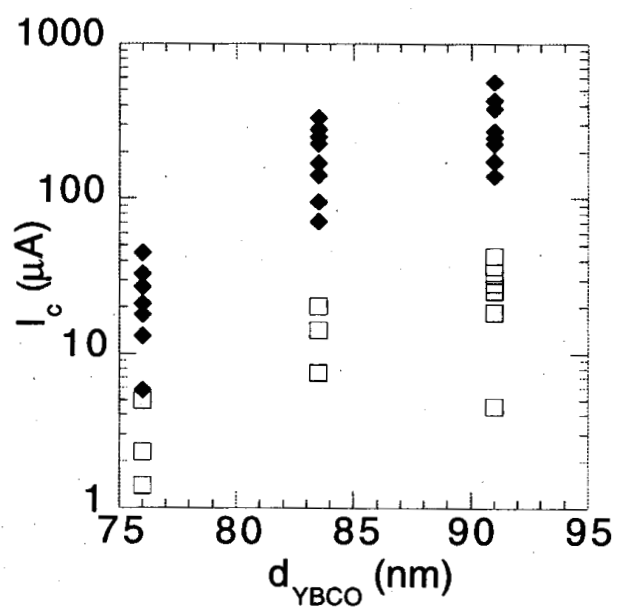

FIG. 3. Dependence of $I_{C}$ on YBCO thickness. The step height was $83 \mathrm{~nm}$; the diamonds are data at $4 \mathrm{~K}$ and the open squares are at $70 \mathrm{~K}$.

We have also obtained results from arrays with identical step heights but with varying YBCO thickness. This experiment uses what in fact is a deficiency in our deposition system: a non-uniform deposited thickness when material is grown at an angle to the laser plume. This is undesirable for large area coverage and is a source of $I_{c}$ variation. If necessary it can be alleviated greatly by oscillating the position of the heater block during growth. We generally cluster our devices within a $2 \mathrm{~mm}$ region of the sample; for the sake of these experiments the arrays were stretched out to the edges of the die. As the YBCO thickness increases, the edge of the film at the bottom of the step approaches the edge of the upper film, in principle reducing the junction length. We can see this effect clearly in the plot of Fig. 3. As the YBCO becomes thicker, the average $I_{C}$ increases. While it is tempting to use the results of Fig. 2 and Fig. 3 to estimate the normal metal coherence length, it is clear that with only three length values one could not make a statistically convincing argument about the value of $\xi_{N}$. In the future, it may be possible to use the thickness gradient over many arrays to obtain enough data that a least-squares fit could be made to the Likharev model.

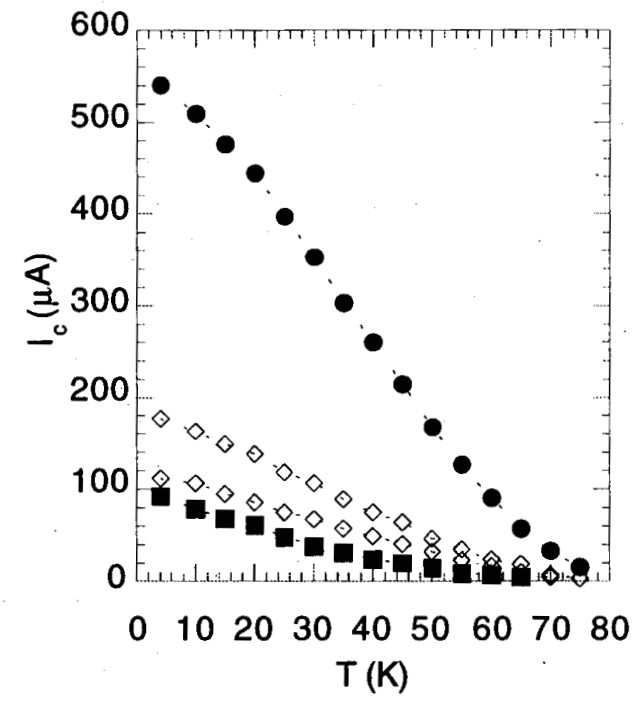

FIG. 4. $I_{c}$ vs. T curves for different junctions on different step heights. The YBCO was $85 \mathrm{~nm}$ thick and the circles are for a 80 $\mathrm{nm}$ step, both sets of diamonds for a $110 \mathrm{~nm}$ step, and the squares for a $140 \mathrm{~nm}$ step.

The temperature dependence of $I_{c}$ also can be used as a qualitative diagnostic of the junction length. Likharev [7] shows a series of curves of $I_{c} R_{n}$ vs. $T / T_{c}$ for various lengths. An inflection point in the curves which moves to lower temperatures as the length increases. This is due to the dominance of the exponential term of Eqn. 1 over the power law dependence on temperature, at low temperatures and/or long lengths. Figure 4 shows $I_{c}(T)$ for 4 different single junctions from the variable length array experiment. We have begun to carry out detailed fits of this temperature dependence [10]. Qualitatively, there is a noticeable inflection point that appears to drop to lower temperature for the bridges with large $d_{\text {step. }}$. At the same time, $I_{c}(4 \mathrm{~K})$ decreases, as we would expect for a longer junction.

The variation of $I_{C}$ with junction width was studied with a mask set incorporating junction arrays ranging from $2-20 \mu \mathrm{m}$ in width. $I_{c}(w)$ is shown in Fig. 5 for two different samples. 
As discussed in [9], self field effects will act to limit the maximum critical current observable even in this geometry of junction. Thus, we would expect that the critical current will no longer rise linearly with width as the junction becomes wider than a few times the effective $\lambda_{J}$. Such behavior is seen in Fig. 5, and implies that the effective Josephson penetration depth is approximately $1-2 \mu \mathrm{m}$. This is much larger than the value predicted by the tunnel junction result as well as by calculations carried out by the authors of [9]. As noted earlier, this calls into question the details of the current distribution both in the banks and in the junction itself.

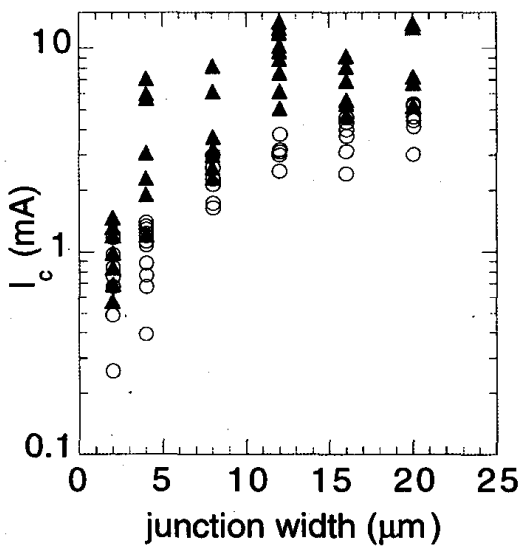

FIG. $5 I_{c}$ vs. junction width for two different samples at $4 \mathrm{~K}$ : triangles are for $d_{y b c o}=85 \mathrm{~nm}$ and $d_{\text {step }}=85 \mathrm{~nm}$, circles are for a sample with $d_{y b c o}=115 \mathrm{~nm}$ and $d_{\text {step }}=125 \mathrm{~nm}$.

\section{B. Dependence of $R_{n}$ on Width and Overlap}

The normal resistance, whether due to interface resistance or to the resistance of the link itself, should vary inversely with width. Scaling with thickness may be much more complex due to film morphology and edge coverage effects. We show data in Fig. 6 with linear fits to individual junctions from the variable width sample discussed above. The excellent agreement with a linear fit shows that $R_{n}$ is behaving as we would expect.

It is important to note that these junctions have an unusual dependence on another geometric factor, the degree of overlap of the Au on both the upper and lower electrodes, $L_{\text {ovlp }}$ (as shown in Fig. 1b). We alluded to this earlier when we stated that $R_{n}$ and $I_{c}$ can be varied independently. The experimental observation is that as $L_{o v l p}$ decreases, $R_{n}$ increases, but $I_{C}$ is relatively unchanged. This is an important and unexpected feature of junction behavior. We can increase $R_{n}$ by as much as a factor of 10 , while $I_{c}$ decreases by approximately $10 \%$. This is a very desirable feature when designing circuits where it may be necessary to have varying $I_{c} R_{n}$ products for different circuit elements. Trimming and adjusting a completed device is also possible; this capability is being used in several experiments now underway.

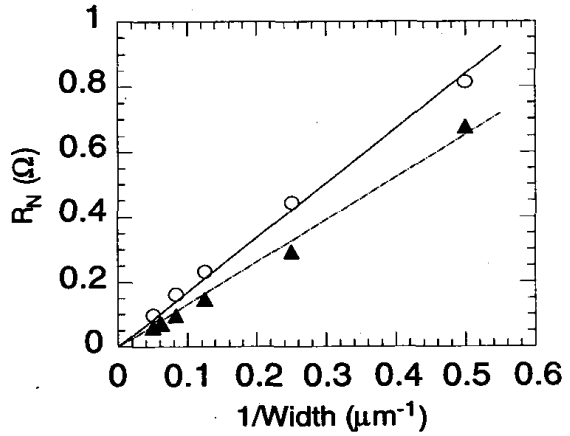

FIG. 6. $R_{n}$ vs. width for the two samples of Fig. 4 , also at $4 \mathrm{~K}$.

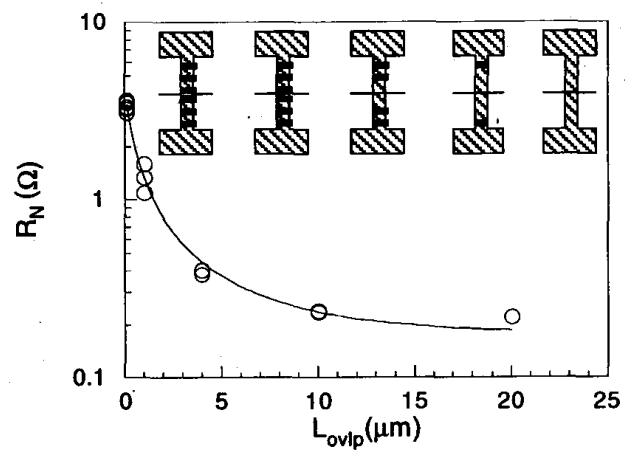

FIG. 7. $R_{n}$ vs. $L_{\text {ovlp }}$ for three junctions which were sequentially cut as shown in the inset. Data were taken at $4 \mathrm{~K}$.

Based on the insensitivity of $I_{c}$ to $L_{o v l p}$, there is no (or very little) supercurrent coupling through the top of the film, while normal current is coupled there. This difference may be due to the anisotropy of epitaxial YBCO films. Thus, most of the measured supercurrent arises from transport through the edge of the film at the exposed $a-b$ planes. A simple model for this anisotropic transport has been developed [11] and is in reasonable agreement with the observed $R_{n}\left(L_{o v l p}\right)$ relationship. The parameters in this model are the boundary resistance at the interface of the overlapped area, the boundary resistance at the exposed " $a-b$ " edge, and the sheet resistance of the Au. Experimentally, we did a series of ion milling processes to sequentially "cut" the $\mathrm{Au}$ on the bilayer surface. After each mill, we measured the junctions' IV characteristics. The results of this for one set of three junctions is shown in Fig. 7; superimposed is a line which is a fit to the model. The inset show how the cuts are brought in closer and closer to the step edge which forms the junction. As in [4], we ended with an angled-ion-mill to reduce the overlap to zero.

The very high boundary resistance observed in these junctions, while possibly limiting our ability to control $I_{C}$ 
spread [5], does allow us to make technologically useful junctions. The usual problem with SNS junctions in the past has been the very low $\boldsymbol{R}_{\boldsymbol{n}}$ values provided by noble metal links. A SINIS junction with inhomogeneous barriers can have large $R_{n}$, while still achieving a very useful level of critical current [10], though perhaps with unacceptably large spreads in $I_{C}$. Exploiting the large $R_{B}$, we have recently fabricated junctions with resistances over $100 \Omega$, and have found that $10 \Omega$ junctions are relatively easy to make reliably.

\section{DISCUSSION OF APPLICATIONS}

We have extended the junction processes described here to multilevel circuits. Preliminary work in this direction has been described in [3]. We are presently working on circuits incorporating arrays of junctions, resonators, antennas, and multiple levels of ground plane and wiring. Work has also recently begun on making steps in $\mathrm{Si}$ and $\mathrm{Si}$-on-sapphire (SOS). This has a two-fold advantage: the Si substrate is known to be very transparent in the IR, and there are advanced techniques for etching $\mathrm{Si}$ in an extremely controlled fashion.

The most promising applications of our junctions are the ones that take advantage of either high $I_{c} R_{n}$ or high $R_{n}$ alone. Detectors, both heterodyne and direct, benefit from large values of $R_{n}$ to match the junction to other (usually high frequency) circuit elements. The paucity of $50 \Omega$ junctions in both low and high temperature superconductors has forced the use of arrays of junctions to match impedances of both sources and amplifiers. Our SNS junctions with $R_{n}$ greater than $30 \Omega$ have been used as $\mathrm{THz}$ detectors and heterodyne mixers with exciting potential for infra-red spectroscopy [12], [13].

If junction uniformity and reproducibility improve, many other avenues open up. One of the most interesting of these is discussed by Benz et al. [14] in building the "programmable" voltage standard [15]. Unfortunately, we do not now have the level of $I_{c}$ control that is needed for applications requiring many junctions. The modest improvements we have seen in control, from a factor of 10 to a factor of 3 in variability, can be attributed to simply more care in the fabrication process. Statistical process control should result in further improvement, as evidenced by the recent results of Murduck et al. [16]. It is an open question as to the degree of control that will be attainable with a reasonable level of effort. Junctions on crystallographically etched features in Si may provide a leap in uniformity. However, the vagaries of edge formation and epitaxial film growth at a break in the surface are obviously reasons to doubt that a step-edge process will be adequate for circuits requiring thousands of junctions, such as the programmable voltage standard. The single junction characteristics are, however, so ideal that we are carrying out conceptual tests of few-junctions circuits and pursuing applications where the unique single junction capabilities are needed.

\section{REFERENCES}

[1] M.S. Dilorio, S. Yoshizumi, K.Y. Yang, J. Zhang, and M. Maung, "Practical high- $\mathrm{T}_{\mathrm{C}}$ Josephson junctions and $\mathrm{dc}$ SQUIDs operating above $85 \mathrm{~K}, "$ Appl. Phys. Lett., vol. 58, pp. 2552-2554, 1991

[2] R.H. Ono, J.A. Beall, M.W. Cromar, T.E. Harvey, M.E Johansson, C.D. Reintsema, and D.A. Rudman, "High-T superconductor-normal metal-superconductor Josephson microbridges with high-resistance normal metal links," Appl. Phys. Lett., vol. 59, pp. 1126-1128, August 1991.

[3] N Missert, T.E. Harvey, R. H. Ono, and C.D. Reintsema "High-Tc multilayer step-edge Josephson junctions and SQUIDs," Appl. Phys. Lett., vol 63, pp. 1690-1692, Sept. 1993.

[4] P.A. Rosenthal, E. N. Grossman, R. H. Ono, and L.R. Vale, Superconductor-Normal Metal-Superconductor Junctions with High Characteristic Voltage," Appl. Phys. Lett., vol. 63, pp. 1984-1986, Oct. 1993.

[5] K. Char, L. Antognazza, and T.Geballe, "Study of interface resistances in epitaxial $\mathrm{YBa}_{2} \mathrm{Cu}_{3} \mathrm{O}_{7-\mathrm{x}} / \mathrm{barrier} / \mathrm{YBa}_{2} \mathrm{Cu}_{3} \mathrm{O}_{7-\mathrm{x}}$ junctions," Appl. Phys. Lett, vol. 63, pp. 2420-2422, Oct 1993.

[6] P.G. DeGennes, "Boundary effects in superconductors," Rev. Mod. Phys., vol. 36, pp225-237, Jan. 1964.

[7] K.K. Likharev, "The relationship $I_{s}(\phi)$ for SNS Junctions," Sov. Tech. Phys. Lett,. vol. 2, pp. 12-13, 1976

[8] A., Kleinsasser and $K$. Delin, "Demonstration of the proximity effect in $\mathrm{YBa}_{2} \mathrm{C}_{3} \mathrm{O}_{7-\mathrm{x}}$ Edge Junctions," unpublished.

[9] R.L. Kautz, S.P. Benz, and C.D. Reintsema, "Largeamplitude Shapiro steps and self-field effects in high-Tc Josephson weak links," Appl. Phys. Lett., vol. 65, pp. 1126-1100, Sept. 1994.

[10] S.J. Berkowitz, L. Antognazza, K. Char, R.H. Ono, N. Missert, P.A. Rosenthal, L.R. Vale, P.M. Mankiewich, and W.J. Skocpol, "Interface scattering in high temperature superconducting-normal-superconducting Josephson junctions," unpublished.

[11] C.D. Reintsema, P.A. Rosenthal, R.H. Ono, L.R. Vale, unpublished.

[12] P.A. Rosenthal and E.N. Grossman, "Terahertz Shapiro steps in high temperature SNS Josephson junctions," IEEE Trans. Microwave Theory and Tech., vol 42, pp. 707-714, April 1994.

[13] E.N. Grossman, L.R. Vale, D.A. Rudman, L.R. Zink, and K.M Evenson, "HTS Josephson junctions as Terahertz mixers: output noise and $30 \mathrm{THz}$ mixing experiments," unpublished.

[14] S.P. Benz, C.D. Reintsema, R.H. Ono, J.Eckstein, I. Bosovic and G. Virshup, "Suitability of high- $T_{c}$ Josephson junctions for voltage standard arrays," unpublished.

[15] C.A. Hamilton, C.J. Burroughs, and R.L. Kautz, "Josephson D/A converter with fundamental accuracy," accepted for publication in IEEE Trans. Instrum. Meas., Sept. 1994.

[16] J.M. Murduck, C. L. Pettiette-Hall, and J.A. Luine, "Improved step-edge junction uniformity and circuit yield," Extended Abstracts of the International Workshop on High Temperature Superconducting Electron Devices, Whistler BC, pp. 126-127, May 1994. 